\title{
Spectra and Elliptic Flow of (Multi)Strange Hadrons at RHIC and LHC within Viscous Hydrodynamics + Hadron Cascade Hybrid Model
}

\author{
Xiangrong $\mathrm{Zhu}^{1,2}$ \\ ${ }^{1}$ School of Science, Huzhou University, Huzhou 313000, China \\ ${ }^{2}$ Department of Physics and State Key Laboratory of Nuclear Physics and Technology, Peking University, Beijing 100871, China \\ Correspondence should be addressed to Xiangrong Zhu; xrongzhu@pku.edu.cn
}

Received 8 March 2016; Accepted 14 August 2016

Academic Editor: Shusu Shi

Copyright ( 92016 Xiangrong Zhu. This is an open access article distributed under the Creative Commons Attribution License, which permits unrestricted use, distribution, and reproduction in any medium, provided the original work is properly cited. The publication of this article was funded by SCOAP ${ }^{3}$.

Using the $(2+1)$-dimensional ultrarelativistic viscous hydrodynamics + hadron cascade, VISHNU, hybrid model, we study the $p_{\mathrm{T}}$-spectra and elliptic flow of $\Lambda, \Xi$, and $\Omega$ in $\mathrm{Au}+\mathrm{Au}$ collisions at $\sqrt{s_{\mathrm{NN}}}=200 \mathrm{GeV}$ and in $\mathrm{Pb}+\mathrm{Pb}$ collisions at $\sqrt{\mathrm{s}_{\mathrm{NN}}}=$ $2.76 \mathrm{TeV}$. Comparing our model results with the data measurements, we find that the VISHNU model gives good descriptions of the measurements of these strange and multistrange hadrons at several centrality classes at RHIC and LHC. Mass ordering of elliptic flow $v_{2}$ among $\pi, K, p, \Lambda, \Xi$, and $\Omega$ are further investigated and discussed at the two collision systems. We find that, at both RHIC and LHC, $v_{2}$ mass ordering among $\pi, K, p$, and $\Omega$ is fairly reproduced within the VISHNU hybrid model, and more improvements are needed to be implemented for well describing $v_{2}$ mass ordering among $p, \Lambda$, and $\Xi$.

\section{Introduction}

Ultrarelativistic heavy-ion collisions at the BNL Relativistic Heavy-Ion Collider (RHIC) and CERN Large Hadron Collider (LHC) are used to produce and study a hot and dense medium consisting of strongly interacting quarks and gluons, namely, Quark-Gluon Plasma (QGP), which is expected to exist in the early stage of the universe, and to understand its properties, such as the equation of state (EoS) and transport coefficients. The hadronic interactions are expected to have less influence on the multistrange hadrons, such as $\Xi$ and $\Omega$, due to their much smaller hadronic cross-sections. Therefore, final observables of these multistrange hadrons are more sensitive to the early (partonic) stage of the collision. In the past few decades, different aspects of strange and multistrange hadrons have been investigated theoretically [1$13]$ and experimentally [14-24].

Anisotropic flow, which is considered as an evidence for the QGP formation, typically displays the collective behavior of the final emitted particles. It can be characterized by the coefficients of the Fourier expansion of the final particle azimuthal distribution defined as [25]

$$
E \frac{d^{3} N}{d^{3} p}=\frac{1}{2 \pi} \frac{d^{2} N}{p_{\mathrm{T}} d p_{\mathrm{T}} d y}\left(1+2 \sum_{n=1}^{\infty} v_{n} \cos \left[n\left(\varphi-\Psi_{n}\right)\right]\right),
$$

where $v_{n}$ is $n$th order anisotropic flow harmonic with its corresponding reaction plane angle $\Psi_{n}$ and $\varphi$ is the azimuthal angle of the final emitted particles. Recently, the anisotropic flow and other soft hadron data of all charged and identified hadrons at the RHIC and LHC have been studied by many groups within the framework of hydrodynamics [13, 2636]. VISHNU is a hybrid model [37] for single-shot simulations of heavy-ion collisions, which connects the $(2+1)$ dimensional viscous hydrodynamics with a hadronic afterburner. Employing the VISHNU hybrid model, the specific QGP shear viscosity values of $(\eta / s)_{\mathrm{QGP}}$ are extracted from the elliptic flow measurements of charged hadrons with MC-KLN initial conditions [29]. With the extracted $(\eta / s)_{\mathrm{QGP}}$, the VISHNU provides good descriptions of the soft hadron data of 
$\pi, K$, and $p$ at the RHIC and LHC [30]. Compared with other hadrons, anisotropy flow of (multi)strange particles is mainly produced in the QGP stage and less contaminated by the subsequent hadronic interactions. Meanwhile, the $p_{\mathrm{T}}$-spectra and elliptic flow for $\Lambda, \Xi$, and $\Omega$ have been measured in the Au + Au collisions at the RHIC [17-20] and $\mathrm{Pb}+\mathrm{Pb}$ collisions at the LHC [21-23]. Therefore, it is timely to systematically study these strange and multistrange hadrons at RHIC and LHC via the VISHNU hybrid model.

In this paper, we investigate the $p_{\mathrm{T}}$-spectra and elliptic flow $v_{2}$ for (multi)strange hadrons in $\mathrm{Au}+\mathrm{Au}$ collisions at $\sqrt{s_{\mathrm{NN}}}=200 \mathrm{GeV}$ and in $\mathrm{Pb}+\mathrm{Pb}$ collisions at $\sqrt{s_{\mathrm{NN}}}=2.76 \mathrm{TeV}$ within the viscous hydrodynamic hybrid model VISHNU. The paper is organized as follows. Section 2 briefly introduces the VISHNU hybrid model and its setup in the calculations. Section 3 compares our VISHNU results in $\mathrm{Au}+\mathrm{Au}$ collisions and $\mathrm{Pb}+\mathrm{Pb}$ collisions with the measurements from the STAR at RHIC and ALICE at LHC, respectively, mainly including $p_{\mathrm{T}}$-spectra and differential elliptic flow for $\Lambda, \Xi$, and $\Omega$. In Section 4 , the mass ordering of elliptic flow among $\pi, K, p$, $\Lambda, \Xi$, and $\Omega$ is studied and discussed at the RHIC and LHC energies. Finally, we summarize our works and give a brief outlook for the future in Section 5.

\section{Setup of the Calculation}

We here give brief descriptions of the inputs and setup of VISHNU calculations for the soft data at the RHIC and LHC energies. The VISHNU [37] hybrid model consists of two parts, which are the $(2+1)$-dimensional ultrarelativistic viscous hydrodynamics VISH2+1 [39, 40] for the expansion of strongly interacting matter QGP and a microscopic hadronic cascade model (UrQMD) [41, 42] for the hadronic evolution. In the calculations a switching temperature $T_{\text {sw }}$ of $165 \mathrm{MeV}$ is set for the transition from the macroscopic to microscopic approaches in VISHNU. This switching temperature value is close to the QCD phase transition temperature [43-46]. We input the equation of state (EoS) s95p-PCE $[47,48]$ for the hydrodynamic evolution above the switching temperature $T_{\text {sw. }}$. The s95p-PCE, which accounts for the chemical freezeout at $T_{\text {chem }}=165 \mathrm{MeV}$, was constructed by combing the lattice QCD data at high temperature with a chemically frozen hadron resonance gas at low temperature.

Following [29, 30], we input MC-KLN initial conditions [49-51] and start the hydrodynamic simulations at $\tau_{0}=$ $0.9 \mathrm{fm} / \mathrm{c}$. For improving computational efficiency, we implement single-shot simulations $[13,29,30,37,38,52]$ with smooth initial entropy density profiles generated by the MC-KLN model. The smooth initial entropy densities are obtained by averaging over a large number of fluctuating entropy density profiles within a specific centrality class. The initial density profiles are initialized with the reaction plane method, which was once used in [29, 30, 38]. Considering the conversion from total initial entropies to final multiplicity of all charged hadrons, we do the centrality selection through the distribution of total initial entropies that are obtained from the event-by-event fluctuating profiles. Such centrality classification was firstly used by Shen et al. in [53], which is more close to the experimental one defined from the measured multiplicity distributions. The normalization factors for the initial entropy densities in $\mathrm{Au}+\mathrm{Au}$ collisions and $\mathrm{Pb}+\mathrm{Pb}$ collisions are, respectively, fixed to reproduce the charged hadron multiplicity density $d N_{\mathrm{ch}} / d \eta$ with $687.4 \pm$ 36.6 at the RHIC [54] and $1601 \pm 60$ at the LHC [55] at most central collisions. The $\lambda$ parameter in the MC-KLN model, which quantifies the gluon saturation scale in the initial gluon distributions [50], is tuned to 0.218 at the RHIC and 0.138 at the LHC for a better description of the centrality dependent multiplicity density for all charged hadrons.

In the VISHNU simulations with MC-KLN initial conditions, we set a value of 0.16 for the QGP specific shear viscosity $(\eta / s)_{\mathrm{QGP}}$. Such combined setting in VISHNU calculations once nicely described the elliptic flow of $\pi, K$, and $p$ in $\mathrm{Au}$ $+\mathrm{Au}$ collisions [52] and $\mathrm{Pb}+\mathrm{Pb}$ collisions [30]. Here, we continue to use it to further study the soft hadron data of strange and multistrange hadrons at both RHIC and LHC. For simplicity of the theoretical calculations, we neglect the bulk viscosity, net-baryon density, and the heat conductivity in the QGP system evolution.

\section{Spectra and Elliptic Flow}

In Figure 1, we present the transverse momentum spectra of hadrons $\Lambda, \Xi$, and $\Omega$ in $\mathrm{Au}+\mathrm{Au}$ collisions at $\sqrt{s_{\mathrm{NN}}}=$ $200 \mathrm{GeV}$ from the VISHNU hybrid model and compare these results with the STAR measurements. We observe that the VISHNU generally describes the $p_{\mathrm{T}}$-spectra $\Xi$ but slightly overestimates the production of $\Omega$ at all centrality classes. Our VISHNU results of $\Lambda$ at $p_{\mathrm{T}}<2 \mathrm{GeV} / \mathrm{c}$ are about $40 \%$ lower than $\Lambda$ from STAR and about $20 \%$ lower than $\bar{\Lambda}$ from STAR. This can be understood from the following. In our calculations, the production of $\Lambda$ is obtained from the original values of strong resonance decays from UrQMD of VISHNU. For the STAR measurements, the $\Lambda$ spectra are corrected for the feed-down of multistrange baryon weak decays (the feed-down contributions to the $\Xi$ spectra from $\Omega$ decays are negligible) [15]. Meanwhile, the STAR $\Lambda$ spectra are not corrected for the feed-down of $\sigma^{0}$ decays from the channel of $\Sigma^{0} \rightarrow \Lambda+\gamma$ (for $\bar{\Lambda}$, the contribution from $\overline{\Sigma^{0}}$ is via the channel of $\left.\overline{\Sigma^{0}} \rightarrow \bar{\Lambda}+\gamma\right)$. At LHC, it was found that the contribution from $\Sigma^{0}$ for $\Lambda$ is about $30 \%$ in VISHNU calculations [38]. Furthermore, we notice that STAR measurements of $\Lambda$ and $\Xi^{-}$are slightly larger than their corresponding antiparticles due to nonzero baryon density at this collision energy. In our calculations, however, zero net-baryon density is used, which leads to the same results between these (multi)strange hadrons and their antiparticle partners.

We also calculate the transverse momentum spectra of $\Lambda$, $\Xi$, and $\Omega$ in $\mathrm{Pb}+\mathrm{Pb}$ collisions at LHC with our VISHNU hybrid model. The calculations, compared with the measurements from the ALICE Collaboration, are presented in Figure 2. For the ALICE measurements, the production differences between these (multi)strange hadrons and their antiparticles are very small due to very small net-baryon density at the LHC energies. It also shows that the VISHNU generally describes the $p_{\mathrm{T}}$-spectra of hadrons $\Lambda, \Xi$, and $\Omega$ at several centrality classes, except for the $60-80 \%$ centrality bin. 


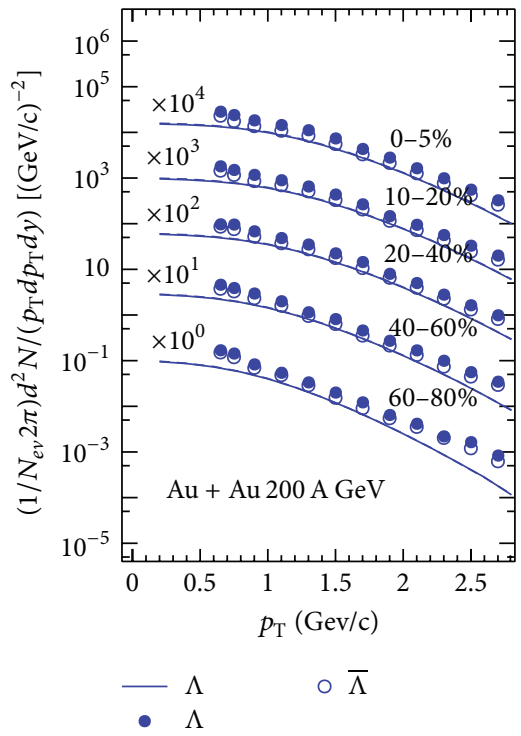

(a)

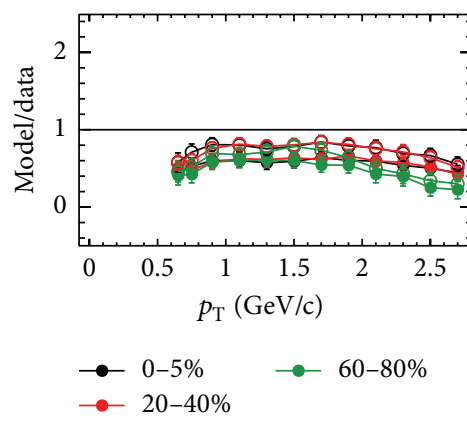

(d)

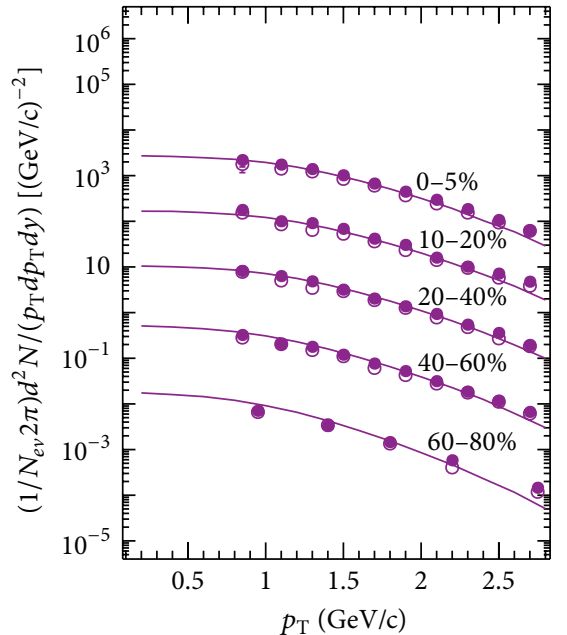

$\circ \bar{\Xi}^{+}$

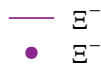

(b)

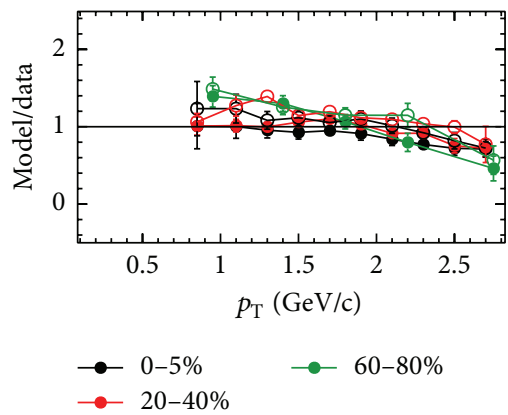

(e)

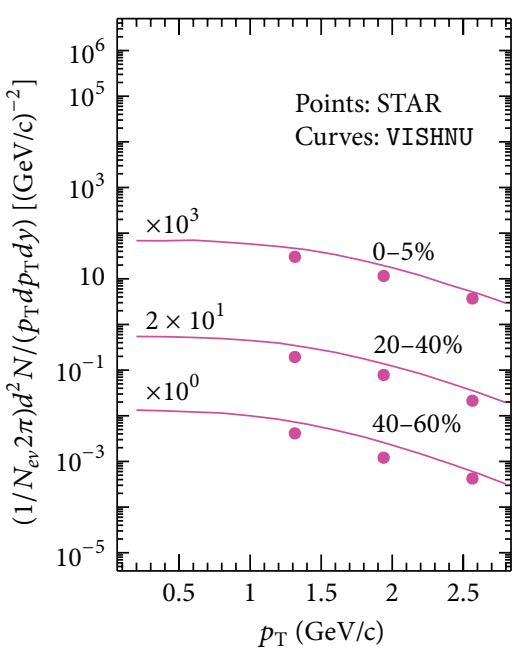

$\Omega^{-}+\bar{\Omega}^{+}$

- $\Omega^{-}+\bar{\Omega}^{+}$

(c)

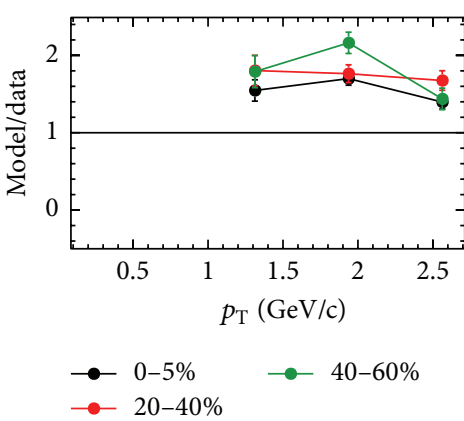

(f)

FIGURE 1: Transverse momentum spectra of $\Lambda(\bar{\Lambda}), \Xi^{-}\left(\bar{\Xi}^{+}\right)$, and $\Omega^{-}+\bar{\Omega}^{+}$at various centralities in Au + Au collisions at $\sqrt{s_{\mathrm{NN}}}=200 \mathrm{GeV}$. Experimental data are taken from STAR measurements [15]. Theoretical curves are calculated by the VISHNU hybrid model with the parameters presented in Section 2. From top to bottom the curves correspond to $0-5 \%\left(\times 10^{4}\right), 10-20 \%\left(\times 10^{3}\right), 20-40 \%\left(\times 10^{2}\right), 40-60 \%\left(\times 10^{1}\right)$, and $60-80 \%\left(\times 10^{0}\right)$ centrality, respectively, where the factors in parentheses are the multipliers applied to the spectra for clear separation. The multiplied factor for spectra of $\Omega$ at $20-40 \%$ is $2 \times 10^{1}$ instead of $1 \times 10^{1}$. In VISHNU, the spectra of particles and corresponding antiparticles are the same due to zero net-baryon density used in our calculations. Therefore, only results of particles are shown with solid curves.

Here, our theoretical calculations of $\Lambda$ only include strong resonance decays from UrQMD of VISHNU. As a result, they are about $30 \%$ lower than the ALICE measurements including contribution from nonweak decays of $\Sigma^{0}$ and $\Sigma(1385)$ family [21]. The $\Omega$ spectra from VISHNU are slightly higher than the experimental data at these centrality classes, as similarly observed in the calculations at the RHIC. Such deviations between theory and experiment are consistent with the model and data differences for the centrality dependent multiplicity shown in [38].

From comparisons between our calculations and measurements at the RHIC and LHC, we find that, although the VISHNU cannot fully reproduce the $p_{\mathrm{T}}$-spectra of these strange and multistrange hadrons in the production amount, it gives nice descriptions of the slopes (distribution shapes) for the spectra of them at various centralities. This can be found from the ratio between model results and data in Figures 1 and 2, which are weakly centrality dependent.
Together with the early nice descriptions of the $p_{\mathrm{T}}$-spectra for $\pi, K$, and $p$ [30], it reveals that during the QGP and hadronic evolution the VISHNU hybrid model generates a proper amount of radial flow to push the spectra of various hadrons.

Figure 3 presents the comparisons of differential elliptic flow of $\Lambda, \Xi$, and $\Omega$ from the VISHNU model with the STAR measurements in $\mathrm{Au}+\mathrm{Au}$ collisions at $\sqrt{s_{\mathrm{NN}}}=200 \mathrm{GeV}$. The theoretical curves are calculated from VISHNU model by using reaction plane initial conditions from the MC-KLN model and $(\eta / s)_{\mathrm{QGP}}=0.16$. The experimental data are from the STAR, which are measured with the event plane method $[19,20]$. This method covers a fraction of contribution from eventby-event flow fluctuations and nonflow contribution mainly including resonance decays and jets. Compared with STAR measurements, the elliptic flow from the VISHNU generally reproduces the data for $\Lambda$ at $0-10 \%$ and $10-40 \%$ and for $\Xi$ and $\Omega$ at $0-30 \%$ centrality classes. At $40-80 \% \mathrm{Au}+\mathrm{Au}$ 


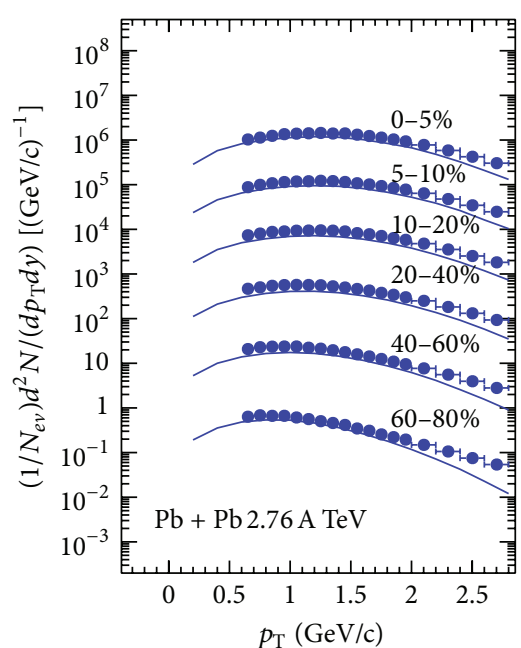

$-\Lambda+\bar{\Lambda}$

(a)

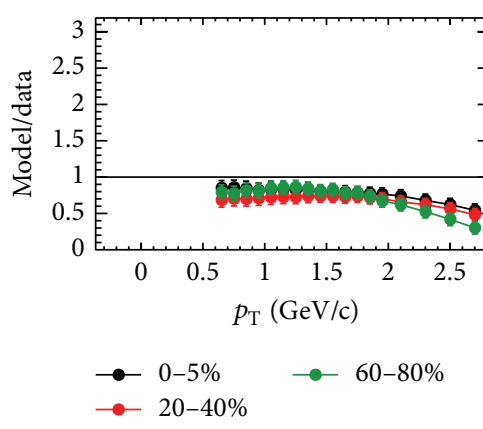

(d)

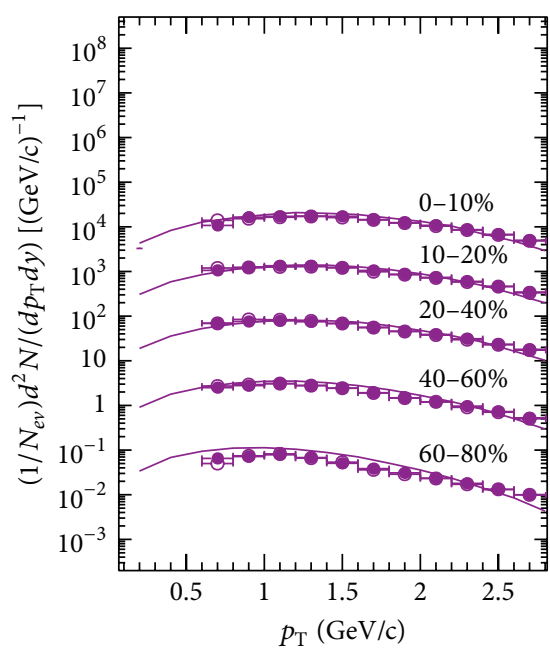

$-\Xi^{-} \quad \circ \bar{\Xi}^{+}$

(b)

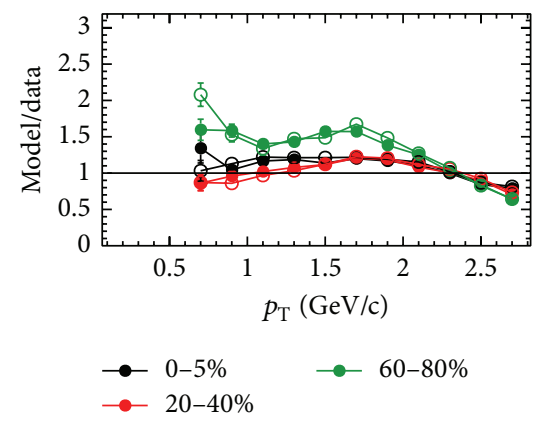

(e)

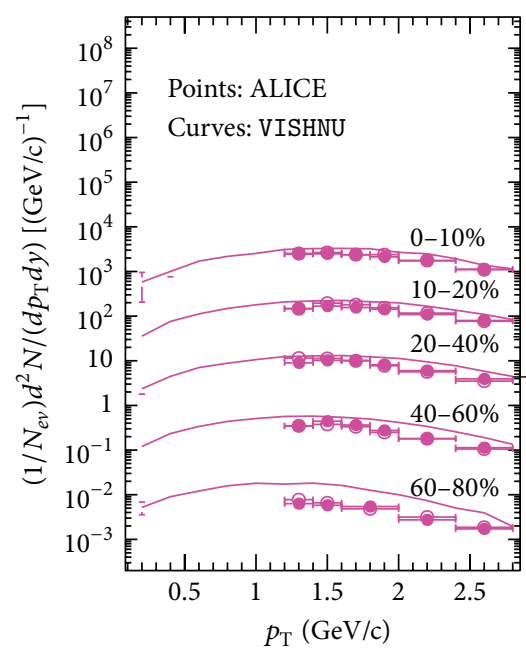

$\Omega^{-} \quad \circ \bar{\Omega}^{+}$

(c)

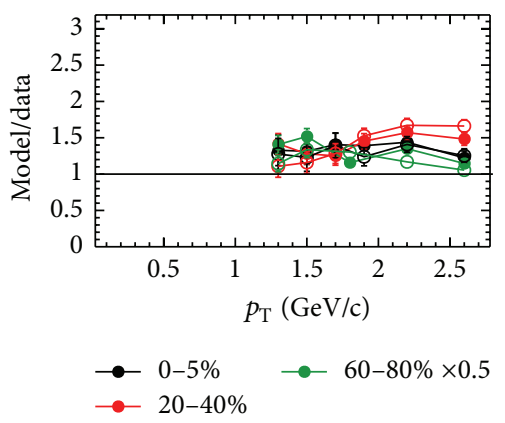

(f)

Figure 2: Transverse momentum spectra of $\Lambda+\bar{\Lambda}, \Xi^{-}\left(\bar{\Xi}^{+}\right)$, and $\Omega^{-}\left(\bar{\Omega}^{+}\right)$at various centralities in $\mathrm{Pb}+\mathrm{Pb}$ collisions at $\sqrt{s_{\mathrm{NN}}}=2.76 \mathrm{TeV}$. Experimental data are taken from LHC $[21,22]$. Theoretical curves are calculated with the VISHNU hybrid model with the parameters presented in Section 2. From top to bottom the curves correspond to $0-10 \%\left(\times 10^{4}\right), 10-20 \%\left(\times 10^{3}\right), 20-40 \%\left(\times 10^{2}\right), 40-60 \%\left(\times 10^{1}\right)$, and $60-80 \%\left(\times 10^{0}\right)$ centrality, respectively, where the factors in parentheses are the multipliers applied to the spectra for clear separation. Spectra of $\Lambda$ start from $0-5 \%\left(\times 10^{5}\right)$ and $5-10 \%\left(\times 10^{4}\right)$, instead of $0-10 \%$. In VISHNU, the spectra of particles and corresponding antiparticles are same due to zero net-baryon density used in our calculations. Therefore, only results of particles are shown with solid curves.

collisions, the model gives rough descriptions of the data for $\Lambda$ at $p_{\mathrm{T}}<1.0 \mathrm{GeV} / \mathrm{c}$ and for $\Xi$ and $\Omega$ at $p_{\mathrm{T}}<1.5 \mathrm{GeV} / \mathrm{c}$ but underestimates at higher $p_{\mathrm{T}}$ region. Together with the failed descriptions of elliptic flow for charged hadrons and identified lighter hadrons at $40-80 \%$ collisions in $[28,29]$, it reflects that the VISHNU, with the MC-KLN initial conditions, fails to describe the $40-80 \%$ semiperipheral collisions. This can be probably interpreted as follows. In $40-80 \%$ collisions, the collision lifetimes are shorter, which leave less time to generate the elliptic flow in the fluid dynamic QGP stage, and the highly dissipative effects in hadronic stage cannot compensate for this.

In Figure 4, we show the differential elliptic flow of $\Lambda, \Xi$, and $\Omega$ at $10-20 \%, 30-40 \%$, and $50-60 \% \mathrm{~Pb}+\mathrm{Pb}$ collisions, which were first given in [38]. The presented experimental data are measured by the ALICE Collaboration with the scalar product method [23]. The VISHNU theoretical results are calculated with the inputs as presented in Section 2. Figure 4 shows that the VISHNU fairly predicts the elliptic flow data for $\Lambda, \Xi$, and $\Omega$ at chosen three centrality classes at $p_{\mathrm{T}}<$ $2 \mathrm{GeV} / c$ within the statistical error bars. At $p_{\mathrm{T}}>2 \mathrm{GeV} / \mathrm{c}$, the descriptions of the elliptic flow for $\Xi$ at $50-60 \%$ and for $\Omega$ at $30-40 \%$ and $50-60 \%$ become worse. Together with the worse descriptions of elliptic flow data at high- $p_{\mathrm{T}}$ at the RHIC, as shown in Figure 3, we consider that the viscous corrections probably become too large at high- $p_{\mathrm{T}}$ region, in which the hydrodynamic description in the VISHNU lost its predictive power.

\section{Mass Ordering of Elliptic Flow}

It is widely accepted that the characteristic mass ordering of differential elliptic flow among various identified hadrons at low- $p_{\mathrm{T}}$ reflects the interplay between radial and elliptic flow, providing more insights into the properties of the QGP fireball. The radial flow creates a depletion in the particle 

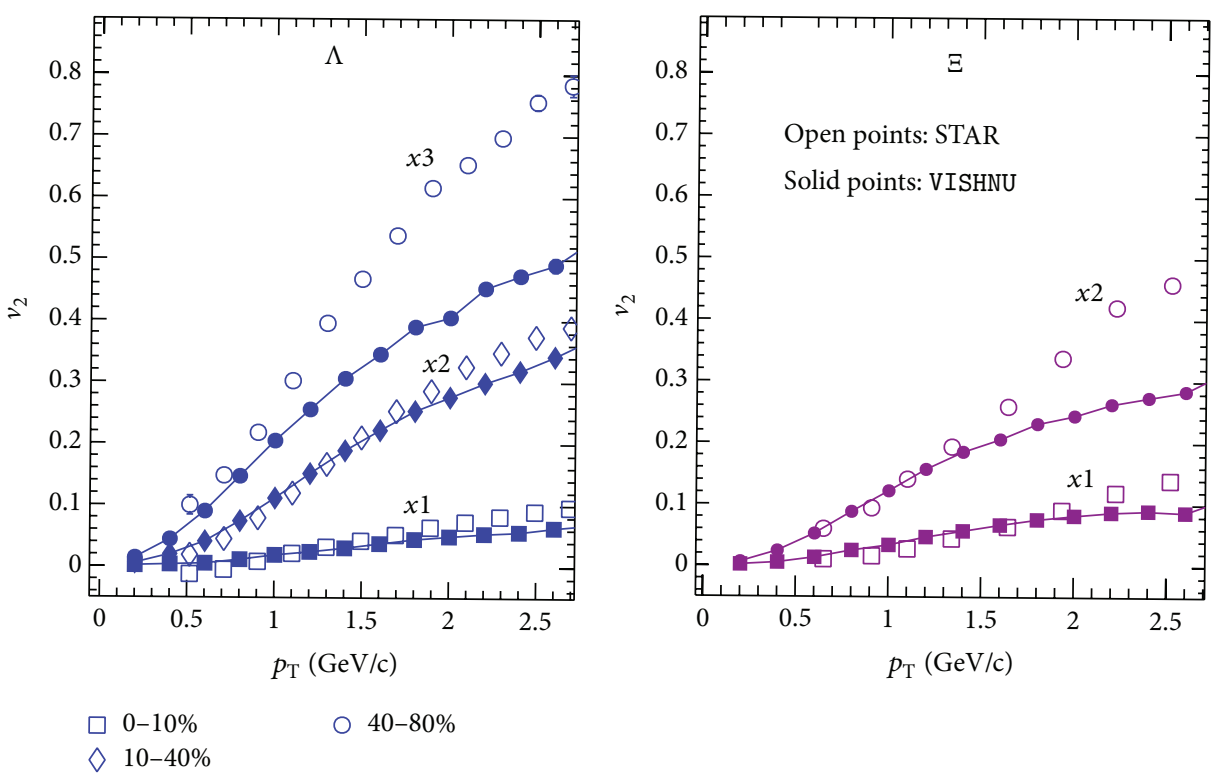

(a)

(b)

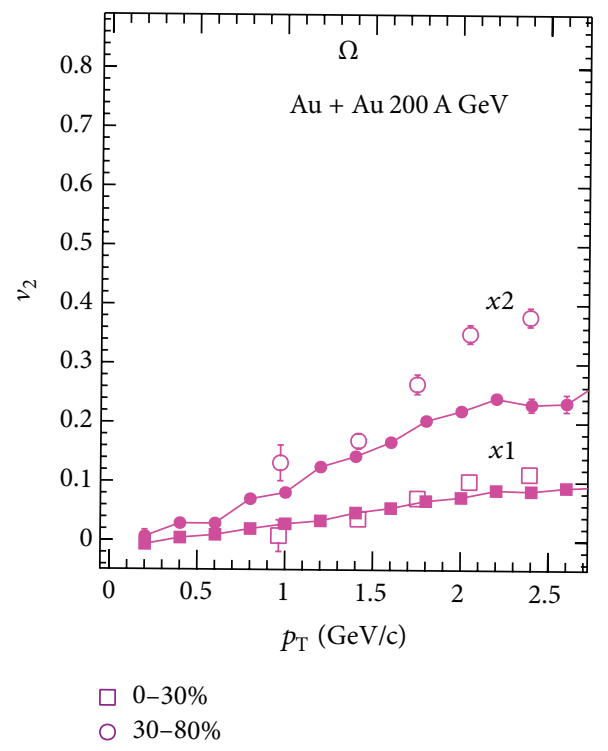

(c)

Figure 3: Differential elliptic flow of strange hadrons $\Lambda$ (a) at centralities of 0-10\% (squares), 10-40\% (diamonds), and 40-80\% (circles), and multistrange hadrons $\Xi$ (b) and $\Omega$ (c) at centralities of $0-30 \%$ (squares) and $30-80 \%$ (circles) in Au + Au collisions at $\sqrt{s_{\mathrm{NN}}}=$ $200 \mathrm{GeV}$. Experimental data (open points) are from STAR $[19,20]$. Theoretical results (solid points) are calculated from the VISHNU viscous hydrodynamics hybrid model with the inputs presented in Section 2.

$p_{\mathrm{T}}$-spectrum at low values, which increases with increasing particle mass. This leads to heavier particles having a smaller $v_{2}$ compared to lighter ones at a given value of $p_{\mathrm{T}}$, giving a mass ordering of the $p_{\mathrm{T}}$ dependent elliptic flow below $1.5-2 \mathrm{GeV} / \mathrm{c}$. Such $v_{2}$ mass ordering has been discovered in the experiments at both the RHIC and LHC [19, 23, 5658], which has also been studied within the framework of hydrodynamics $[8,13,59-61]$ and blastwave model $[8,62]$.

Here we investigate the mass ordering of the elliptic flow among various identified hadrons $\pi, K, p, \Lambda, \Xi$, and $\Omega$ in $\mathrm{Au}+$ $\mathrm{Au}$ collisions and $\mathrm{Pb}+\mathrm{Pb}$ collisions. For clear presentations, the experimental data and our VISHNU results are plotted in separate panels at $0-80 \%$ at the RHIC and $30-40 \%$ at the LHC. Together with the calculations of elliptic flow of identified hadrons in $[29,30]$ and in this paper, we find that the VISHNU generally describes the $v_{2}\left(p_{\mathrm{T}}\right)$ for different identified hadrons at several centrality classes. However, our theoretical results presented in Figure 5, compared with the measurements at RHIC and LHC, show that the VISHNU fairly describes the mass ordering among $\pi, K, p$, and $\Omega$. But it is hard to see mass ordering among $p, \Lambda$, and $\Xi$ clearly due to their elliptic flow being almost identical at 

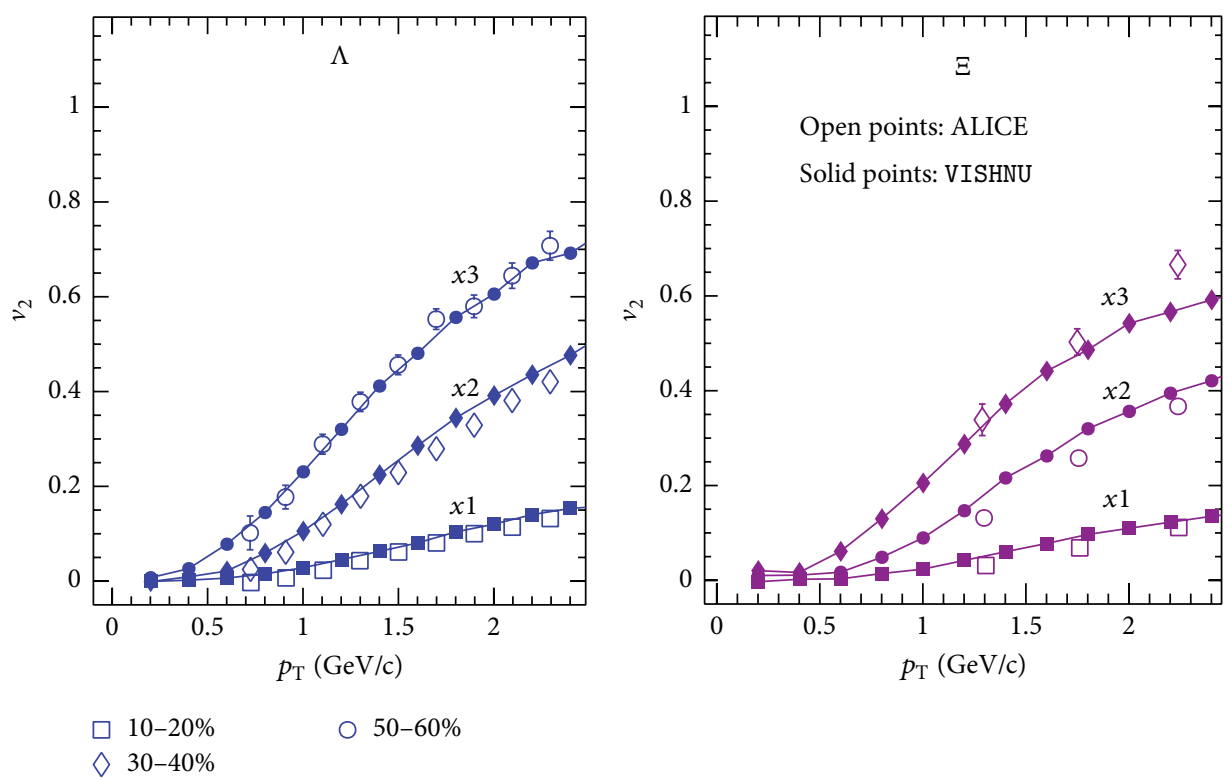

(a)

(b)

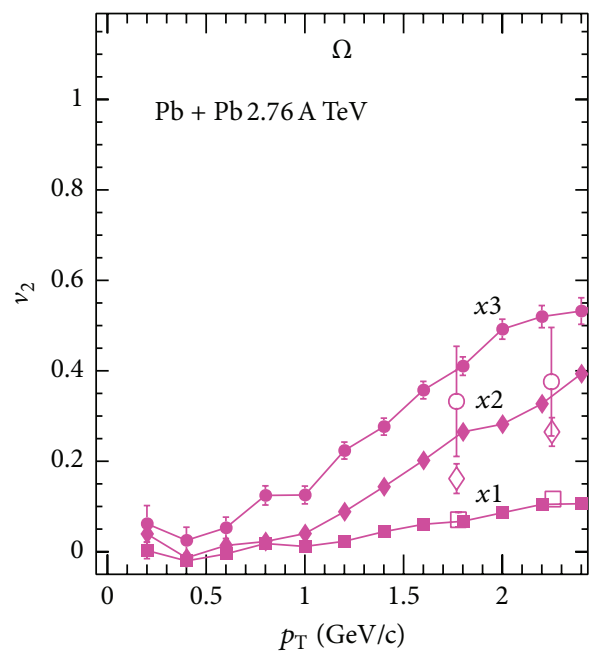

(c)

FIGURE 4: Differential elliptic flow of strange hadrons $\Lambda$ (a), multistrange hadrons $\Xi$ (b) and $\Omega$ (c) at 10-20\% (squares), 30-40\% (diamonds), and $50-60 \%$ (circles) centralities in $\mathrm{Pb}+\mathrm{Pb}$ collisions at $\sqrt{s_{\mathrm{NN}}}=2.76 \mathrm{TeV}$. Experimental data (open points) are from the ALICE measurements [23]. Theoretical results (solid points) are calculated from the VISHNU hybrid model with the inputs presented in Section 2, which were first shown in [38]

low $p_{\mathrm{T}}$ region. This underprediction for proton leads to an inverse $v_{2}$ mass ordering between $p$ and $\Lambda$. Effects of hadronic rescattering on elliptic flow are seen to be particle specific, depending on their scattering cross-sections that couple them to the medium [59]. Compared with nonstrange hadrons, the multistrange hadrons are less affected on their differential elliptic flow due to their smaller scattering cross-sections. Therefore, reevaluating the hadronic cross-sections in the UrQMD is helpful to improve the description of elliptic flow of various hadron species. Meanwhile, an initial flow could enhance the radial flow in the hadronic stage, which is also expected to improve the description of mass ordering within the framework of the hybrid model.

\section{Summary and Outlook}

In summary, we studied the $p_{\mathrm{T}}$-spectra and elliptic flow of strange and multistrange hadrons in $\mathrm{Au}+\mathrm{Au}$ and $\mathrm{Pb}+\mathrm{Pb}$ collisions within the VISHNU hybrid model. At both collision systems, we found that, with MC-KLN initial conditions, $\eta / s=$ 0.16 , and other inputs, VISHNU generally describes the $p_{\mathrm{T}^{-}}$ spectra of strange hadron $\Lambda$ and multistrange hadrons $\Xi$ at some centrality classes but slightly overestimates for $\Omega$ at chosen centrality classes. In spite of the normalization issues, the VISHNU well produces the spectra slopes of these three hadrons at chosen centralities. By comparing the elliptic flow of $\Lambda, \Xi$, and $\Omega$ in $\mathrm{Au}+\mathrm{Au}$ collisions and $\mathrm{Pb}+\mathrm{Pb}$ 


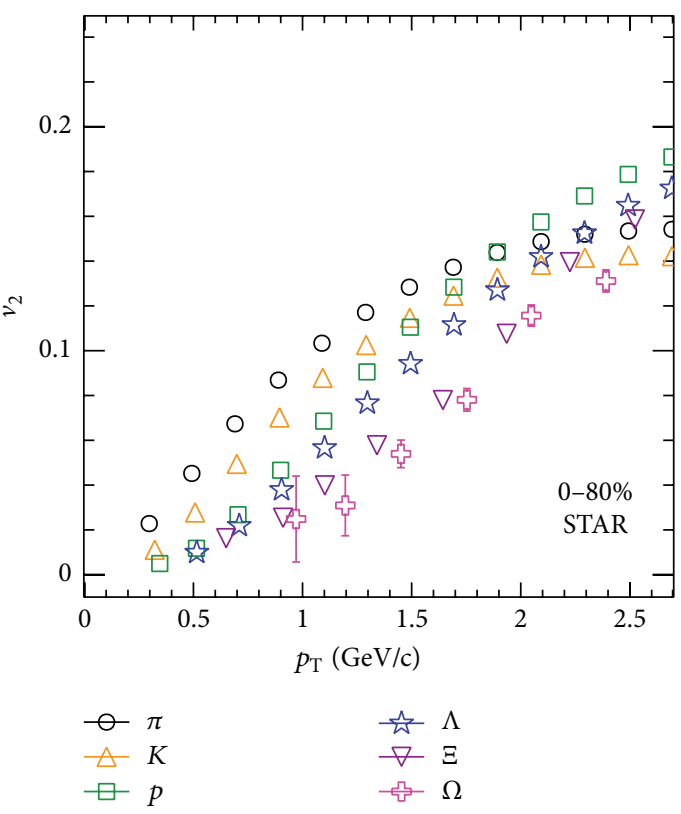

(a)

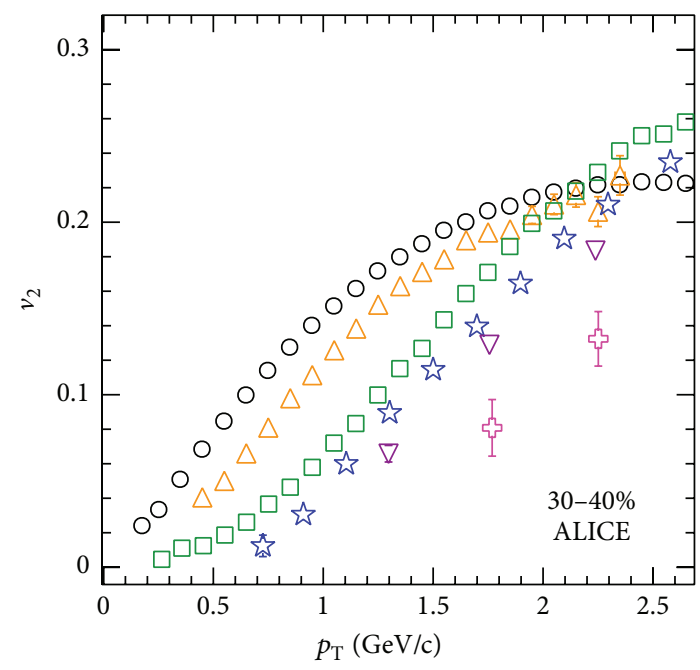

(c)

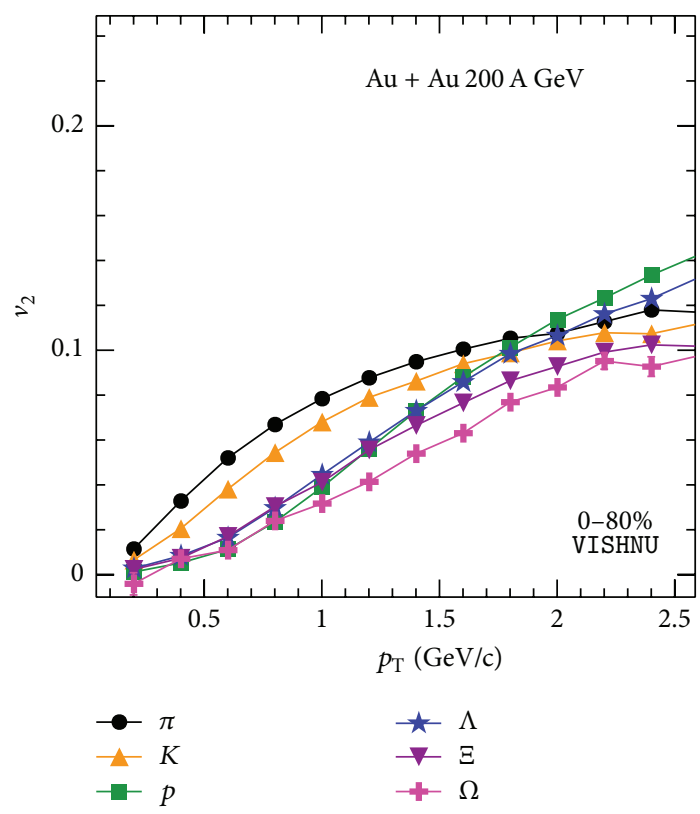

(b)

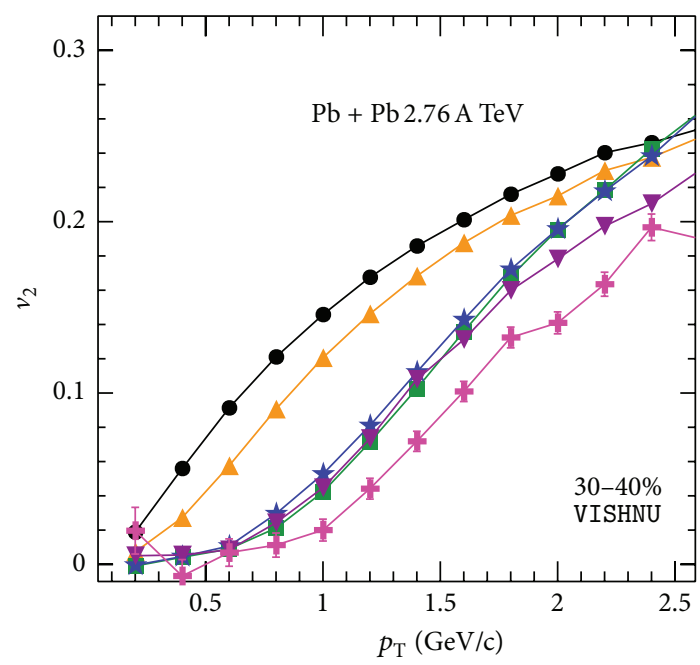

(d)

Figure 5: Differential elliptic flow of $\pi, K, p, \Lambda, \Xi$, and $\Omega$ at centrality $0-80 \%$ in Au + Au collisions at $\sqrt{s_{\mathrm{NN}}}=200 \mathrm{GeV}$ ((a-b), (a) data, (b) VISHNU calculations), and at centrality $30-40 \%$ in $\mathrm{Pb}+\mathrm{Pb}$ collisions at $\sqrt{s_{\mathrm{NN}}}=2.76 \mathrm{TeV}$ ((c-d), (c) data, (d) VISHNU calculations). The measurements of elliptic flow of various hadron species are taken from STAR [15, 20] and ALICE [23].

collisions from VISHNU model with the STAR and ALICE measurements, we found that the VISHNU generally describes the elliptic flow except at $40-80 \%$ semiperipheral collisions. The failed descriptions at $40-80 \%$ collisions is probably due to shorter lifetimes of these collisions, which leave less time to generate the elliptic flow in the fluid dynamic QGP stage.

We also compared the mass ordering of $v_{2}$ among hadrons $\pi, K, p, \Lambda, \Xi$, and $\Omega$ from VISHNU calculations with the STAR and ALICE measurements. The comparisons showed that the elliptic flow mass ordering among various hadron species is not fully described at both RHIC and LHC. The VISHNU fairly describes the mass ordering of $v_{2}$ among $\pi, K, p$, and $\Omega$ but fails to reproduce the mass ordering among $p, \Lambda$, and $\Xi$ due to slight underpredictions of the elliptic flow of protons. The effects from the initial flow and/or improved UrQMD hadronic cross-sections may solve this issue within the framework of VISHNU, which should be investigated in the near future.

\section{Competing Interests}

The author declares that there is no conflict of interests regarding the publication of this paper.

\section{Acknowledgments}

The author gratefully thanks Huichao Song and Hao-jie Xu for fruitful discussions and critical reading of the draft. This 
work was supported in part by the NSFC and the MOST under Grants no. 11435001 and no. 2015CB856900 and the China Postdoctoral Science Foundation under Grant no. 2015M570878. The author especially acknowledges extensive computing resources provided by Tianhe-1A from the National Supercomputing Center in Tianjin, China.

\section{References}

[1] H. Van Hecke, H. Sorge, and N. Xu, "Evidence of early multistrange hadron freeze-out in high energy nuclear collisions," Physical Review Letters, vol. 81, no. 26, pp. 5764-5767, 1998.

[2] J. Rafelski and B. Müller, "Strangeness production in the QuarkGluon plasma," Physical Review Letters, vol. 48, article 1066, 1982, Erratum-ibid, vol. 56, article 2334, 1986.

[3] S. Hamieh, K. Redlich, and A. Tounsi, "Canonical description of strangeness enhancement from $\mathrm{p}-\mathrm{A}$ to $\mathrm{Pb}-\mathrm{Pb}$ collisions," Physics Letters B, vol. 486, no. 1-2, pp. 61-66, 2000.

[4] J. Letessier and J. Rafelski, "Observing quark-gluon plasma with strange hadrons," International Journal of Modern Physics E, vol. 9, no. 2, p. 107, 2000.

[5] G. Torrieri and J. Rafelski, "Search for QGP and thermal freezeout of strange hadrons," New Journal of Physics, vol. 3, p. 12, 2001.

[6] U. W. Heinz, "Strange messages: chemical and thermal freezeout in nuclear collisions," Journal of Physics G: Nuclear and Particle Physics, vol. 25, no. 2, pp. 263-274, 1999.

[7] G. Torrieri and J. Rafelski, "Strange hadron resonances as a signature of freeze-out dynamics," Physics Letters B, vol. 509, no. 3-4, pp. 239-245, 2001.

[8] P. Huovinen, P. F. Kolb, U. W. Heinz, P. V. Ruuskanen, and S. A. Voloshin, "Radial and elliptic flow at RHIC: further predictions," Physics Letters B, vol. 503, no. 1-2, pp. 58-64, 2001.

[9] V. K. Tiwari and C. P. Singh, "Strangeness enhancement-a potential signature for QGP phase," Physics Letters B, vol. 411, no. 1-2, pp. 225-229, 1997.

[10] C. Blume and C. Markert, "Strange hadron production in heavy ion collisions from SPS to RHIC," Progress in Particle and Nuclear Physics, vol. 66, no. 4, pp. 687-880, 2011.

[11] N. K. Behera, R. Sahoo, and B. K. Nandi, "Constituent quark scaling of strangeness enhancement in heavy-ion collisions," Advances in High Energy Physics, vol. 2013, Article ID 273248, 11 pages, 2013.

[12] A. Bazavov, H.-T. Ding, P. Hegde et al., "Additional strange hadrons from QCD thermodynamics and strangeness freezeout in heavy ion collisions," Physical Review Letters, vol. 113, no. 7, Article ID 072001, 5 pages, 2014.

[13] X. Zhu and H. Song, "Spectra and elliptic flow for $\Lambda, \Xi$, and $\Omega$ in $200 \mathrm{~A} \mathrm{GeV} \mathrm{Au+Au} \mathrm{collisions,"} \mathrm{Journal} \mathrm{of} \mathrm{Physics:} \mathrm{Conference}$ Series, vol. 668, Article ID 012080, 2016.

[14] J. Adams, C. Adler, M. M. Aggarwal et al., "Multistrange baryon production in Au-Au collisions at $\sqrt{s_{N N}}=130 \mathrm{GeV}$," Physical Review Letters, vol. 92, no. 18, Article ID 182301, 6 pages, 2004.

[15] J. Adams, M. M. Aggarwal, Z. Ahammed et al., "Scaling properties of hyperon production in $\mathrm{Au}+\mathrm{Au}$ Collisions at $\sqrt{s_{N N}}=$ 200 GeV," Physical Review Letters, vol. 98, no. 6, Article ID 062301, 2007.

[16] B. I. Abelev, M. M. Aggarwal, Z. Ahammed et al., "Enhanced strange baryon production in $\mathrm{Au}+\mathrm{Au}$ collisions compared to $\mathrm{p}+$ $p$ at $\sqrt{s_{N N}}=200 \mathrm{GeV}$," Physical Review C, vol. 77, Article ID 044908, 2008.
[17] M. M. Aggarwal, Z. Ahammed, A. V. Alakhverdyants et al., "Strange and multistrange particle production in $\mathrm{Au}+\mathrm{Au}$ collisions at $\sqrt{s_{N N}}=62.4 \mathrm{GeV}$," Physical Review $C$, vol. 83, no. 2, Article ID 024901, 16 pages, 2011.

[18] J. Adams, M. M. Aggarwal, Z. Ahammed et al., "Multistrange Baryon elliptic flow in Au+Au collisions at $\sqrt{s_{N N}}=200 \mathrm{GeV}$," Physical Review Letters, vol. 95, Article ID 122301, 2005.

[19] B. I. Abelev, M. M. Aggarwal, Z. Ahammed et al., "Centrality dependence of charged hadron and strange hadron elliptic flow from $\sqrt{s_{N N}}=200 \mathrm{GeV}$ Au+Au collisions," Physical Review C, vol. 77, Article ID 054901, 2008.

[20] L. Adamczyk, J. K. Adkins, G. Agakishiev et al., "Centrality and transverse momentum dependence of elliptic flow of multistrange hadrons and $\phi$ meson in $\mathrm{Au}+\mathrm{Au}$ collisions at $\sqrt{s_{N N}}=200 \mathrm{GeV}$," Physical Review Letters, vol. 116, Article ID 062301, 2016.

[21] B. B. Abelev, J. Adam, D. Adamová et al., " $K_{S}^{0}$ and $\Lambda$ production in $\mathrm{Pb}-\mathrm{Pb}$ collisions at $\sqrt{{s_{N N}}_{1}}=2.76 \mathrm{TeV}$," Physical Review Letters, vol. 111, no. 22, Article ID 222301, 10 pages, 2013.

[22] B. B. Abelev, J. Adam, D. Adamová et al., "Multi-strange baryon production at mid-rapidity in $\mathrm{Pb}-\mathrm{Pb}$ collisions at $\sqrt{s_{N N}}=$ 2.76 Tev," Physics Letters B, vol. 728, pp. 216-227, 2014, Corrigendum to Physics Letters B, vol. 734, pp. 409-410, 2014.

[23] B. B. Abelev, J. Adam, D. Adamová et al., "Elliptic flow of identified hadrons in $\mathrm{Pb}-\mathrm{Pb}$ collisions at $\sqrt{s_{N N}}=2.76 \mathrm{TeV}$," Journal of High Energy Physics, vol. 2015, no. 6, article 190, 2015.

[24] Md. Nasim, V. Bairathi, M. K. Sharma, B. Mohanty, and A. Bhasin, "A review on $\phi$ meson production in heavy-ion collision," Advances in High Energy Physics, vol. 2015, Article ID 197930, 16 pages, 2015.

[25] S. Voloshin and Y. Zhang, "Flow study in relativistic nuclear collisions by Fourier expansion of azimuthal particle distributions," Zeitschrift fur Physik C-Particles and Fields, vol. 70, no. 4, pp. 665-671, 1996.

[26] B. Schenke, S. Jeon, and C. Gale, "Anisotropic flow in $\sqrt{s}=$ 2.76 TeV $\mathrm{Pb}+\mathrm{Pb}$ collisions at the LHC," Physics Letters B, vol. 702, no. 1, pp. 59-63, 2011.

[27] Z. Qiu, C. Shen, and U. Heinz, "Hydrodynamic elliptic and triangular flow in $\mathrm{Pb}-\mathrm{Pb}$ collisions at $\sqrt{s_{\mathrm{NN}}}=2.76 A \mathrm{Tev}$," Physics Letters B, vol. 707, no. 1, pp. 151-155, 2012.

[28] H. Song, S. A. Bass, U. Heinz, T. Hirano, and C. Shen, "Hadron spectra and elliptic flow for $200 \mathrm{~A} \mathrm{GeV} \mathrm{Au+Au} \mathrm{collisions} \mathrm{from}$ viscous hydrodynamics coupled to a Boltzmann cascade," Physical Review C, vol. 86, Article ID 054910, 2011, Erratum: Physical Review C, vol. 86, Article ID 059903, 2012.

[29] H. Song, S. A. Bass, and U. Heinz, "Elliptic flow in $\sqrt{s}=$ $200 \mathrm{GeV} \mathrm{Au}+\mathrm{Au}$ collisions and $\sqrt{s}=2.76 \mathrm{TeV} \mathrm{Pb}+\mathrm{Pb}$ collisions: insights from viscous hydrodynamics+hadron cascade hybrid model," Physical Review C, vol. 83, Article ID 054912, 2011, Erratum: Physical Review C, vol. 87, Article ID 019902, 2013.

[30] H. Song, S. A. Bass, and U. W. Heinz, "Spectra and elliptic flow for identified hadrons in $2.76 \mathrm{~A} \mathrm{TeV} \mathrm{Pb}+\mathrm{Pb}$ collisions," Physical Review C, vol. 89, no. 3, Article ID 034919, 9 pages, 2014.

[31] H. Song, "QGP viscosity at RHIC and the LHC-a 2012 status report," Nuclear Physics A, vol. 904-905, pp. 114c-121c, 2013.

[32] H. Petersen, J. Steinheimer, G. Burau, and M. Bleicher, "Elliptic flow in an integrated $(3+1) \mathrm{d}$ microscopic + macroscopic approach with fluctuating initial conditions," European Physical Journal C, vol. 62, no. 1, pp. 31-36, 2009.

[33] T. Hirano, U. W. Heinz, D. Kharzeev, R. Lacey, and Y. Nara, "Hadronic dissipative effects on elliptic flow in ultrarelativistic 
heavy-ion collisions," Physics Letters B, vol. 636, no. 6, pp. 299304, 2006.

[34] D. Teaney, J. Lauret, and E. V. Shuryak, "Flow at the SPS and RHIC as a quark-gluon plasma signature," Physical Review Letters, vol. 86, no. 21, pp. 4783-4786, 2001.

[35] P. Bozek, "Components of the elliptic flow in $\mathrm{Pb}-\mathrm{Pb}$ collisions at $\sqrt{s}=2.76$ TeV," Physics Letters B, vol. 699, no. 4, pp. 283-286, 2011.

[36] S. Takeuchi, K. Murase, T. Hirano, P. Huovinen, and Y. Nara, "Effects of hadronic rescattering on multistrange hadrons in high-energy nuclear collisions," Physical Review C-Nuclear Physics, vol. 92, no. 4, Article ID 044907, 2015.

[37] H. Song, S. A. Bass, and U. Heinz, "Viscous QCD matter in a hybrid hydrodynamic+Boltzmann approach," Physical Review C, vol. 83, Article ID 024912, 2011.

[38] X. Zhu, F. Meng, H. Song, and Y. X. Liu, "Hybrid model approach for strange and multistrange hadrons in $2.76 \mathrm{~A} \mathrm{TeV}$ $\mathrm{Pb}+\mathrm{Pb}$ collisions," Physical Review C, vol. 91, no. 3, Article ID 034904, 2015.

[39] H. Song and U. Heinz, "Suppression of elliptic flow in a minimally viscous quark-gluon plasma," Physics Letters B, vol. 658, no. 5, pp. 279-283, 2008.

[40] H. Song and U. Heinz, "Causal viscous hydrodynamics in $2+1$ dimensions for relativistic heavy-ion collisions," Physical Review C, vol. 77, no. 6, Article ID 064901, 25 pages, 2008.

[41] S. A. Bass, M. Belkacem, M. Bleicher et al., "Microscopic models for ultrarelativistic heavy ion collisions," Progress in Particle and Nuclear Physics, vol. 41, pp. 255-369, 1998.

[42] M. Bleicher, E. Zabrodin, C. Spieles et al., "Relativistic hadronhadron collisions in the ultra-relativistic quantum molecular dynamics model," Journal of Physics G, vol. 25, no. 9, article 1859, 1999.

[43] Y. Aoki, Z. Fodor, S. D. Katz, and K. K. Szabó, “The QCD transition temperature: results with physical masses in the continuum limit," Physics Letters B, vol. 643, no. 1, pp. 46-54, 2006.

[44] Y. Aoki, S. Borsányi, S. Dürr et al., "The QCD transition temperature: results with physical masses in the continuum limit II," Journal of High Energy Physics (JHEP), vol. 906, p. 88, 2009.

[45] S. Borsányi, Z. Fodor, C. Hoelbling et al., "Is there still any $T_{c}$ mystery in lattice QCD? Results with physical masses in the continuum limit III," Journal of High Energy Physics, vol. 2010, article 73, 2010.

[46] A. Bazavov, T. Bhattacharya, M. Cheng et al., "Chiral and deconfinement aspects of the QCD transition," Physical Review $D$, vol. 85, Article ID 054503, 2012.

[47] P. Huovinen and P. Petreczky, "QCD equation of state and hadron resonance gas," Nuclear Physics A, vol. 837, no. 1-2, pp. 26-53, 2010.

[48] C. Shen, U. Heinz, P. Huovinen, and H. Song, "Systematic parameter study of hadron spectra and elliptic flow from viscous hydrodynamic simulations of $\mathrm{Au}+\mathrm{Au}$ collisions at $\sqrt{s_{\mathrm{NN}}}=$ 200 Gev," Physical Review C, vol. 82, no. 5, Article ID 054904, 13 pages, 2010.

[49] A. Adil, H.-J. Drescher, A. Dumitru, A. Hayashigaki, and Y. Nara, "Eccentricity in heavy-ion collisions from color glass condensate initial conditions," Physical Review C, vol. 74, no. 4, Article ID 044905, 2006.

[50] H. J. Drescher and Y. Nara, "Effects of fluctuations on the initial eccentricity from the color glass condensate in heavy ion collisions," Physical Review C, vol. 75, Article ID 034905, 2007.
[51] H. J. Drescher and Y. Nara, "Eccentricity fluctuations from the color glass condensate in ultrarelativistic heavy ion collisions," Physical Review C, vol. 76, Article ID 041903, 2007.

[52] H. Song, S. A. Bass, U. Heinz, T. Hirano, and C. Shen, "200 A GeV Au+Au collisions serve a nearly perfect QuarkGluon liquid," Physical Review Letters, vol. 106, no. 19, Article ID 192301, 4 pages, 2011, Erratum to Physical Review Letters, vol. 109, no. 13, Article ID 139904, p. 1, 2012.

[53] C. Shen, Z. Qiu, H. Song, J. Bernhard, S. Bass, and U. Heinz, "The iEBE-VISHNU code package for relativistic heavy-ion collisions," Computer Physics Communications, vol. 199, pp. 6185, 2016.

[54] A. Adare, M. Makek, L. Zou et al., "Transverse energy production and charged-particle multiplicity at midrapidity in various systems from $\sqrt{s_{N N}}=7.7$ to $200 \mathrm{GeV}$," Physical Review $C$, vol. 93, no. 2, Article ID 024901, 2016.

[55] K. Aamodt, A. A. Quintana, A. M. Adare et al., "Centrality dependence of the charged-particle multiplicity density at midrapidity in $\mathrm{Pb}-\mathrm{Pb}$ collisions at $\sqrt{s_{N N}}=2.76 \mathrm{TeV}$,' Physical Review Letters, vol. 106, no. 3, Article ID 032301, 10 pages, 2011.

[56] J. Adams, M. M. Aggarwal, Z. Ahammed et al., "Azimuthal anisotropy in $\mathrm{Au}+\mathrm{Au}$ collisions at $\sqrt{s_{N N}}=200 \mathrm{GeV}$," Physical Review C, vol. 72, no. 1, Article ID 014904, 23 pages, 2005.

[57] M. Issah and A. Taranenko, "Scaling characteristics of azimuthal anisotropy at RHIC," http://arxiv.org/abs/nucl-ex/ 0604011.

[58] R. Snellings, "How much does the hadronic phase contribute to the observed anisotropic flow at the LHC?" https:// arxiv.org/abs/1411.7690.

[59] T. Hirano, U. Heinz, D. Kharzeev, R. Lacey, and Y. Nara, "Mass ordering of differential elliptic flow and its violation for mesons," Physical Review C-Nuclear Physics, vol. 77, no. 4, Article ID 044909, 2008.

[60] P. Bozek, "Modeling global event properties using hydrodynamics from RHIC to LHC," AIP Conference Proceedings, vol. 1422, pp. 34-43, 2012.

[61] H. Song, F. Meng, X. Xin, and Y. X. Liu, "Elliptic flow of $\Lambda$, $\Xi$ and $\Omega$ in $2.76 \mathrm{~A} \mathrm{TeV} \mathrm{Pb}+\mathrm{Pb}$ collisions," Journal of Physics: Conference Series, vol. 509, Article ID 012089, 2014.

[62] C. Adler, Z. Ahammed, C. Allgower et al., "Identified particle elliptic flow in $A u+A u$ collisions at $\sqrt{s_{\mathrm{NN}}}=130 \mathrm{GeV}$," Physical Review Letters, vol. 87, Article ID 182301, 2001. 

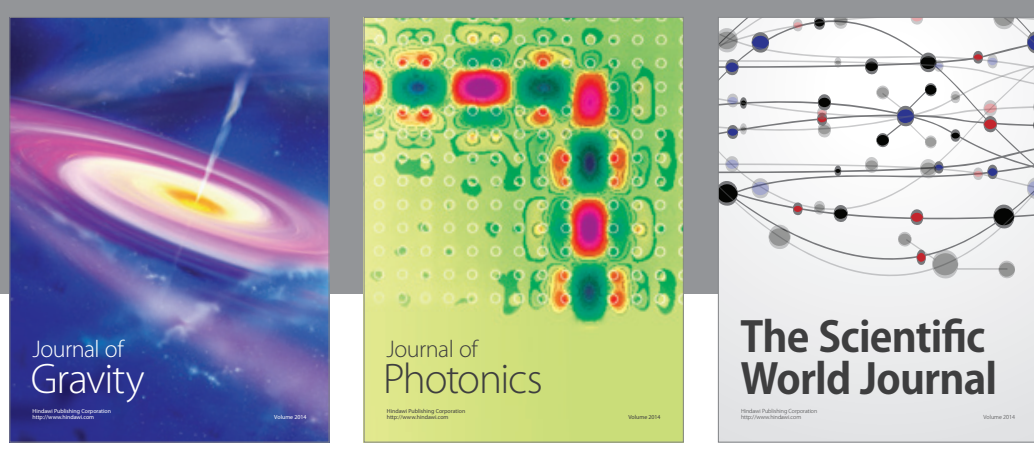

The Scientific World Journal
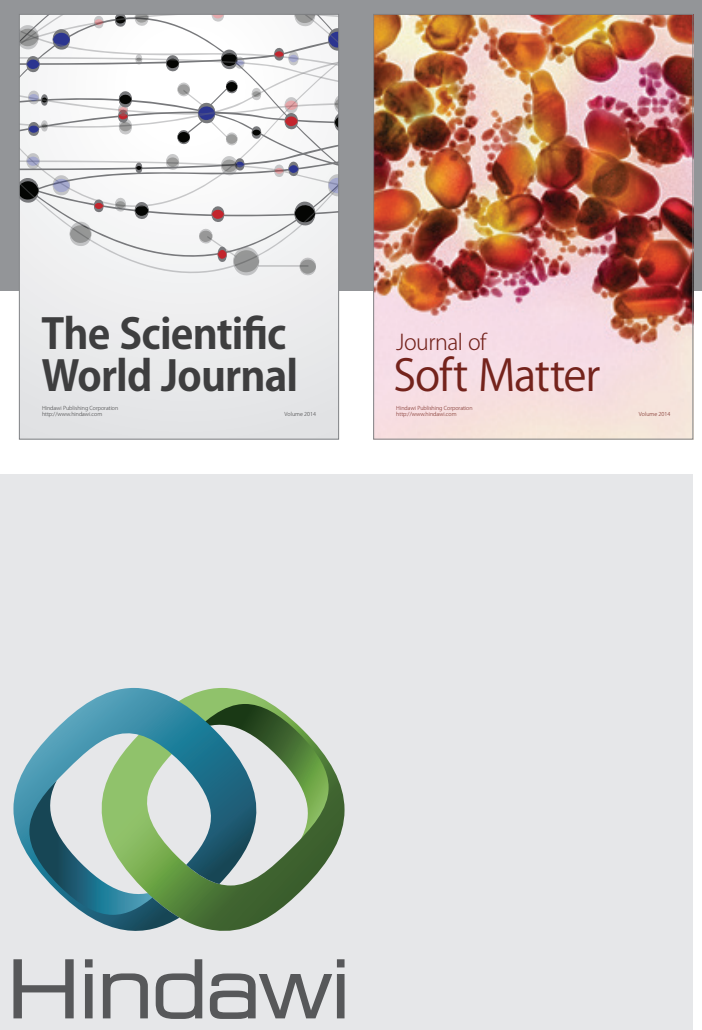

Submit your manuscripts at

http://www.hindawi.com

nternational Journal of

Statistical Mechanics
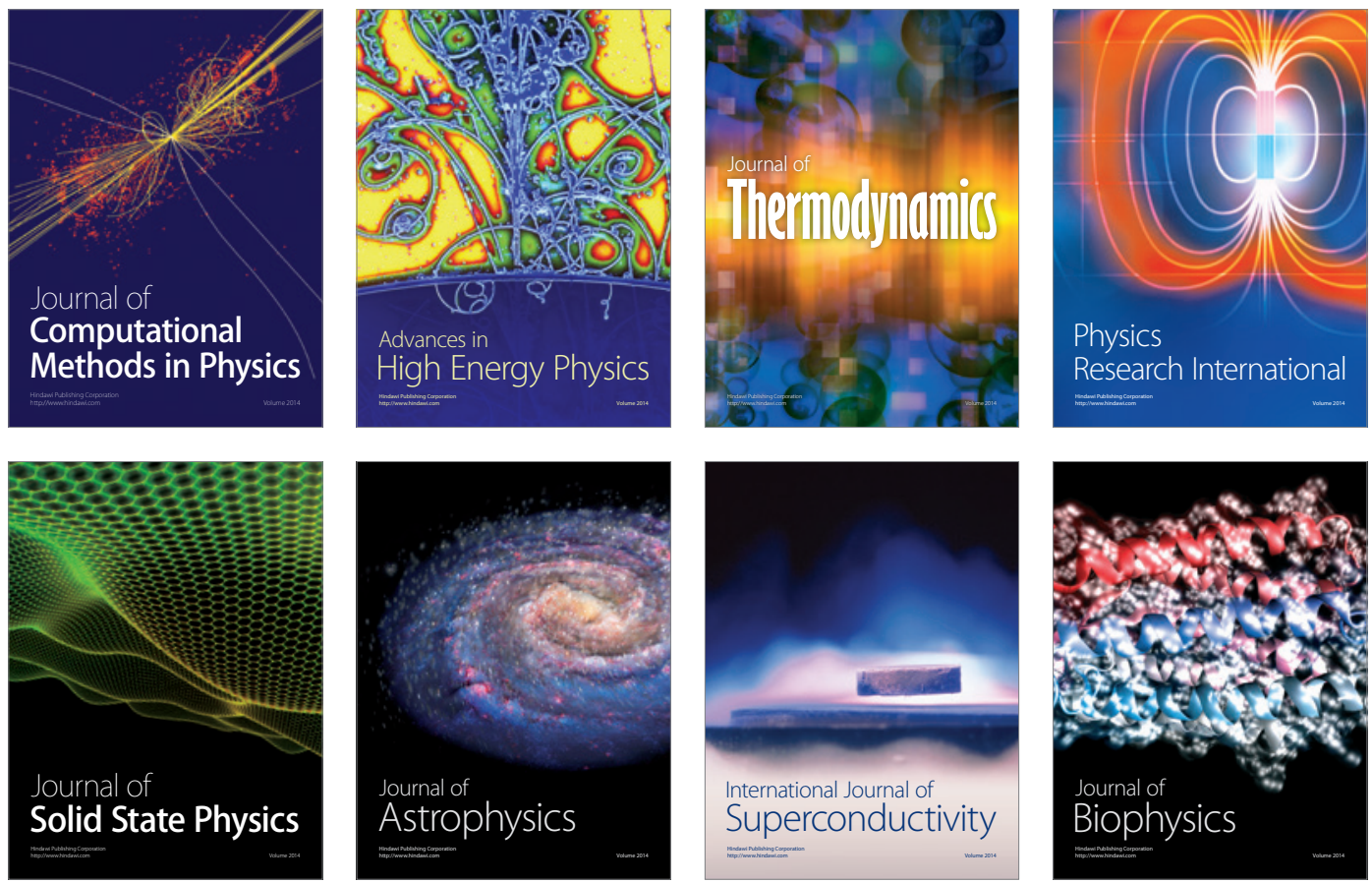
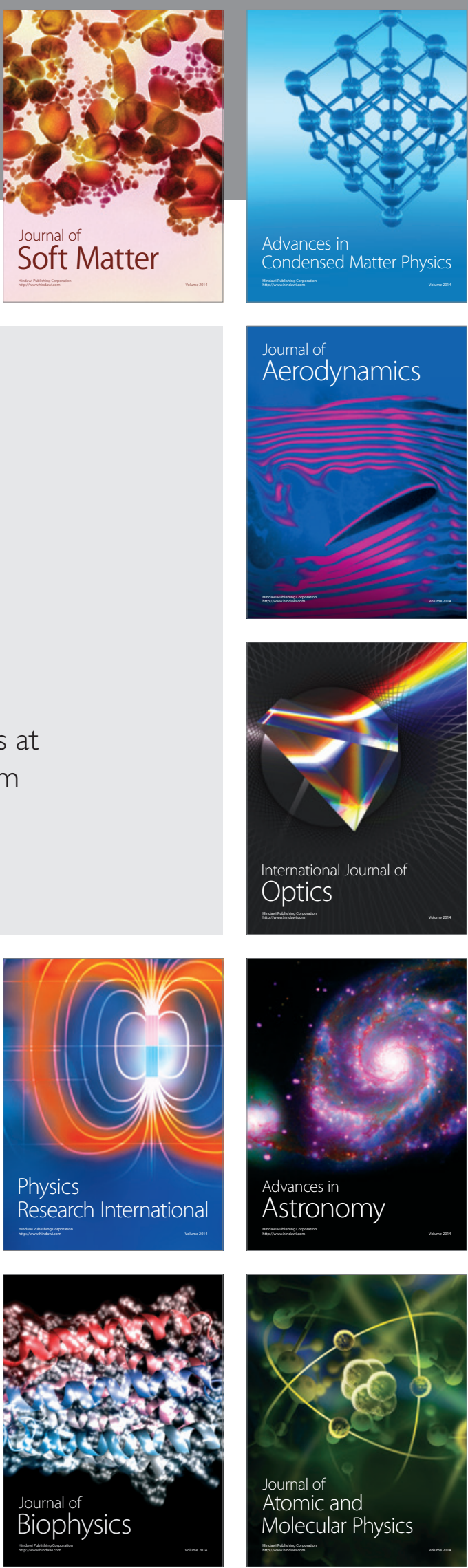